\title{
Galaxy cluster cosmology from X-ray surveys of the hot and energetic Universe
}

\author{
Nicolas Clerc ${ }^{1}$, Barbara Sartoris ${ }^{2,3}$, Klaus Dolag ${ }^{4}$, Rukmani \\ Vijayaraghavan $^{5}$ and Veronica Biffi ${ }^{2,3}$ \\ ${ }^{1}$ Max-Planck Institut für extraterrestrische Physik, \\ Postfach 1312, D-85741 Garching bei München, Germany \\ email: nclerc@mpe.mpg.de \\ ${ }^{2}$ Dipartimento di Fisica, Sezione di Astronomia, Universitá di Trieste, \\ Via Tiepolo 11, I-34143 Trieste, Italy \\ ${ }^{3}$ INAF/Osservatorio Astronomico di Trieste, Via Tiepolo 11, I-34143 Trieste, Italy \\ ${ }^{4}$ Universitäts-Sternwarte München, Scheinerstrasse 1, 81679 München, Germany \\ ${ }^{5}$ Department of Astronomy, University of Virginia \\ 530 McCormick Road Charlottesville, VA 22904, USA
}

\begin{abstract}
We discuss recent advances and prospects in our understanding of the formation of structures on cosmic scales based on surveys of galaxy clusters in the X-ray bands. We highlight the interaction between observations and numerical simulations of the X-ray sky. We show how future surveys will unveil the nature of the dark energy and study its evolution with time.
\end{abstract}

Keywords. Cosmology: observations, cosmology: theory, large-scale structure of universe, galaxies: clusters: general, X-rays: galaxies: clusters, surveys

\section{Introduction}

Clusters of galaxies are excellent tracers of the formation of structures on cosmological scales and probe the matter-energy content of the Universe. The use of galaxy clusters for precision cosmology requires large samples and the understanding of the physical link between their mass and observational properties. Large surveys of the extragalactic sky in X-rays are indispensable to ensuring their efficient detection and their characterization. Indeed, the diffuse intergalactic baryonic matter being heated up to plasma temperatures $\left(10^{7}-10^{8} \mathrm{~K}\right)$ as it is captured by the deepest gravitational wells in the Universe $\left(10^{13}-10^{15} \mathrm{M}_{\odot}\right)$ emits X-ray photons in enormous quantities, that scale with cluster mass. Successful cosmological exploitation of a galaxy cluster survey in X-rays relies in particular on large observed areas with adequate depths and angular resolutions, on the accurate calibration of the mass-observable relations and sample selection biases, on suitable observational follow-up strategies and on targeted comparisons between cosmological models, simulations and observations. Such requirements motivate the development of wide-area surveys of the sky in X-rays and detailed numerical simulations of the large-scale structure.

Our contributions highlight the role of galaxy clusters in the context of cosmological $\mathrm{X}$-ray surveys. An overview of recent results is presented along with first results from the XMM-XXL survey (Sect.2). We then describe the Magneticum Pathfinder suite of simulations, aiming at reproducing the X-ray Universe on very large scales, and a series of detailed simulations describing the fate of circumgalactic coronae in clusters and groups (Sect. 3). Our conclusion is dedicated to future prospects (Sect.4). 


\section{Cosmology with clusters and the role of X-ray surveys}

In the last twenty years, a combination of different observational probes, such as the type-Ia supernovae (SNIa, e.g. Riess et al. 1998, Perlmutter et al. 1999, Rest et al. 2014) the Cosmic Microwave Background (CMB) anisotropies (e.g. Planck Collaboration 2015a), the large scale structure (LSS, e.g. Samushia et al. 2014, Mantz et al. 2015a), have set the current standard cosmological model $(\Lambda \mathrm{CDM})$. In such scenario the Universe is undergoing a phase of accelerated expansion: understanding its source and properties is one principal aim of modern cosmology. The $\Lambda \mathrm{CDM}$ model explains the accelerated expansion by a form of dark energy (DE) that constitutes $\sim 70 \%$ of the Universe energymatter content, with a constant equation of state (EoS), expressed as $w=P / \rho=-1$. A number of models with different forms for the DE EoS have been proposed, as well as models explaining the cosmic acceleration by a modification of the energy-momentum tensor in the Einstein equations (e.g. Amendola, 2000; Caldwell \& Kamionkowski, 2009 ; Ratra \& Peebles, 1988; Tsujikawa, 2010). Alternatives to dark energy include suitable modifications of General Relativity (e.g. Hu \& Sawicki, 2007 ; Sotiriou \& Faraoni, 2010 ; Dvali et al. 2000, Movahed et al. 2009 ; Skordis 2009).

\subsection{Galaxy clusters as cosmological probes}

Clusters of galaxies have played an important role in the construction of the current scenario (e.g. Allen et al. 2011), since they probe at once the growth of structure and the expansion history of the Universe. Therefore they distinguish among different nonstandard models that mimic the $\Lambda \mathrm{CDM}$ expansion history, but have a peculiar growth of structures (e.g. Albrecht et al. 2006). Clusters can be used as cosmological probes through a variety of methods:

- the number density of clusters in a given redshift and mass bin is exponentially sensitive to the amount of matter in the Universe $\left(\Omega_{m}\right)$ and to the normalization of the power spectrum $\sigma_{8}$ (e.g. Eke et al. 1998, Borgani et al. 2001, Pierpaoli et al. 2001)

- the redshift evolution of the mass function constrains the linear growth rate of density perturbations, and thus the DE EoS and its evolution (e.g. Vikhlinin et al. 2009c).

- the spatial distribution of clusters (i.e., correlation function and power spectrum) provides direct information on the underlying cosmological model in many ways:

$\circ$ predictions from inflationary models on the index $n_{s}$ of the initial power spectrum can be verified ( $n_{s}=1$ for the Harrison-Zel'dovich spectrum in the standard model), ○ the shape of the underlying Dark Matter (DM) power spectrum over a broad range of wavenumbers disentangles DM scenarios (Cold DM, Hot DM, etc.) that show a different cut-off on the power spectrum shape (e.g. Costanzi et al. 2013),

$\circ$ the transfer function contains information on the wavenumber corresponding to the comoving scale of the Hubble radius at the epoch of equivalence $\left(k_{e q}\right)$,

o similarly as in the galaxy distribution, Baryonic Acoustic Oscillations (BAOs) are present in the power spectrum of clusters ; the position and amplitude of the wiggles are related to the amount of DM and baryons (e.g. Sartoris et al. 2012),

$\circ$ the ratio $b(M)$ - the bias of DM halos - between the perturbation in the number density of clusters of mass $M$ and the perturbation amplitude of the matter density, as well as redshift-space distortions, are again sensitive to the growth rate of perturbations (e.g. Sheth \& Tormen 1999).

- the baryon fraction in clusters constrains the density parameter, once the cosmic baryon density parameter is known. Tracing the baryon fraction of distant clusters provides a geometrical test of cosmic expansion (e.g. Allen et al. 2008, Ettori et al. 2009). 
- clusters considered as rare objects can test specific cosmological models (e.g. Mortonson et al. 2011). Possible tensions with $\Lambda$ CDM model have been reported after the discoveries of distant $(z>1$ ), massive, clusters (Mullis et al. 2005; Rosati et al. 2009 ; Brodwin et al. 2010). Such tests require precise estimates of the mass, as well as good calibration of the theoretical mass function in a regime challenging simulations (Tinker et al. 2008).

\subsection{Cosmological constraints obtained with $X$-ray cluster surveys}

The role of $X$-ray surveys. Observations in X-rays are key to cluster cosmology studies, both from an observational point of view - clusters show as extended continuous objects on sky, projection effects are minimized -, and from a modeling point of view - selections are tractable and cluster masses scale with X-ray observables. Pencil-beam surveys realized with Chandra probe groups and distant clusters, while surveys covering a fraction of the entire sky (mainly originating from $R O S A T$ ) are able to detect the most massive systems in the nearby Universe. XMM-Newton with its large grasp (effective area $\times$ field-of-view) covers wide areas and captures $M^{*}$ systems at high redshift.

Cosmological constraints and the mass-observable link. Earlier studies (e.g. Eke et al. 1998; Viana \& Liddle 1999; Henry 2000; Pierpaoli et al. 2001; Borgani et al. 2001 ; Seljak 2002 ; Schuecker et al. 2003; Bahcall \& Bode 2003; Voevodkin \& Vikhlinin 2004) used the cluster mass function to constrain $\Omega_{m}$ and $\sigma_{8}$, but were limited by small sample sizes and imprecise estimations of the cluster masses (e.g. through the X-ray flux.)

Using a large sample of ROSAT clusters extending to $z \sim 0.9$ (the 400d, Burenin et al. 2007) and accurate mass proxies from Chandra follow-up observations, Vikhlinin et al. (2009b) improved the reliability of the $M_{t o t}$ vs. proxy relations and confirmed the theoretical results from Tinker et al. (2008) by estimating the cluster mass functions at low and high redshifts. Vikhlinin et al. (2009c) constrained cosmological parameters using the same sample, in particular the DE EoS parameter, $w=-1.14 \pm 0.21$, demonstrating the importance of combining the cluster information with those obtained from CMB, SNIa and BAOs.

Henry et al. (2009) measured the X-ray temperature distribution of local clusters and set tight constraints on the values of $\Omega_{m}$ and $\sigma_{8}$, relying on an internal $L_{X}-T$ relation and on several calibrations of the mass-temperature scaling, including numerical simulations folded through X-ray simulators. Böhringer et al. (2014) used a flux-limited sample of $\sim 800$ clusters from the Southern ROSAT all-sky survey to derive their luminosity function up to $z=0.4$. The cosmological parameters $\Omega_{m}$ (at $14 \%$ level) and $\sigma_{8}(9 \%)$ were constrained using a mass-luminosity relation, whose uncertain knowledge represents the main source of systematics in this study.

Mantz et al. (2014) used the cluster sample of Mantz et al. (2015b) to constrain cosmological parameters from measurements of the gas fraction. By incorporating a robust gravitational lensing calibration of the X-ray mass estimates, constraints were derived on $\Omega_{m}(0.29 \pm 0.04)$ in the $\Lambda \mathrm{CDM}$ model, and on the DE $\operatorname{EoS}(w=-0.98 \pm 0.26)$ in the $w$-CDM model. Taking advantage of a large redshift range in the sample, the evolution of the DE EoS (in the case $w=w_{0}+w_{a}(1+z)$ ) was probed with $w_{0}=-1.7 \pm 1.0$ and $w_{a}=2.2_{-2.7}^{+2.4}$.

An illustration of the importance of the observable-mass calibration for obtaining tight cosmological constraints is given in Mantz et al. (2015a, their Fig. 1) by comparing results on $\left(\Omega_{m}, \sigma_{8}\right)$ obtained in three studies. These studies rely on nearly identical samples ( $\sim 200$ systems), but differ in the way cluster masses are estimated. The most recent of them uses gravitational lensing data, able to calibrate the mass at $\sim 8 \%$ level and to place constraints on $\Lambda \mathrm{CDM}$ parameters at $\Omega_{m}=0.26 \pm 0.03$ and $\sigma_{8}=0.83 \pm 0.04$, improving by a factor of two the precision obtained by previous work. 

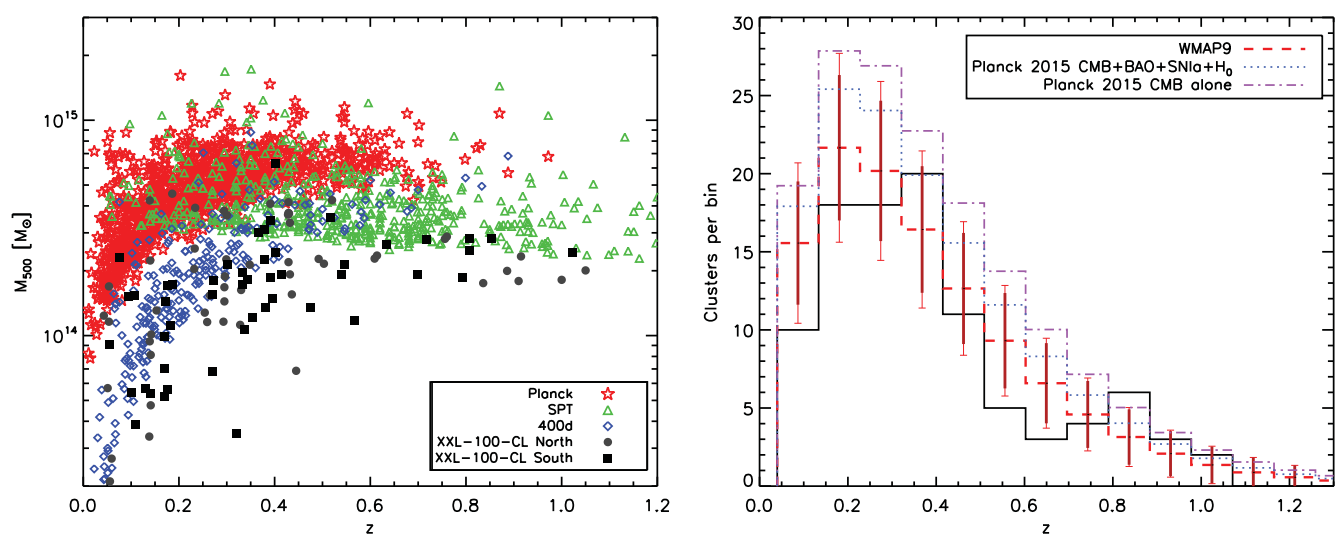

Figure 1. The distribution of the 100 brightest $X M M-X X L$ galaxy clusters (from Pacaud et al. submitted to A\&A). Left: the lower-mass and higher-redshift location of XMM clusters in comparison with recent samples (Burenin et al. 2007, Vikhlinin et al. 2009b, Bleem et al. 2015, Planck Collaboration et al. 2015b). Right: the observed cluster redshift distribution (plain histogram) is compared to predictions from several cosmological models (Hinshaw et al. 2013, Planck Collaboration et al. 2015a) with error bars accounting for shot noise and cosmic variance.

\subsection{The XMM-XXL survey, a deep-wide area cosmological survey}

The XMM-XXL survey (Pierre et al. submitted to A\&A) consists in two fields of $25 \mathrm{deg}^{2}$ each paved with $10 \mathrm{ks} X M M$ observations and is designed to discover $\sim 450$ galaxy clusters up to $z \gtrsim 1$. The cosmological exploitation of the $X M M$-XXL survey will rely on the full sample of galaxy clusters by combining a performant X-ray cluster search, a dedicated follow-up strategy (imaging, spectroscopy, multi- $\lambda$ ) and a thorough simulation programme. Its study will combine the mass function and its evolution (up to $z \sim 1$ ) and the large-scale structure formed by groups and clusters within the probed volume, hence providing standalone constraints on fundamental cosmological models, particularly regarding the DE EoS.

The first XMM-XXL results highlight a golden sample made of its 100 brightest galaxy clusters, defined such as their soft X-ray flux within a 1 arcmin aperture exceeds $3 \times 10^{-14}$ ergs.s ${ }^{-1} . \mathrm{cm}^{-2}$. Their X-ray properties (luminosity $L_{X}$, temperature $T_{X}$ ) are converted into halo mass estimates, after calibration by gravitational shear measurements from the CFHT-LenS weak-lensing data (Lieu et al. A\&A in press). Their $L_{X}-T_{X}$ relation and its evolution in redshift is carefully studied, accounting for selection biases (Giles et al. A\&A in press). Fig. 1 (left) shows the complementarity of XXL-100 clusters to recent cluster surveys in the mass-redshift plane (Pacaud et al. A\&A in press). Knowledge of the cluster selection function and the redshift completeness of the sample allow the cosmological modeling of the sample (Fig. 1, right), accounting for the calibration of the mass-observable relation from the survey (Giles et al. A\&A in press, Lieu et al. A\&A in press). A preference is found for a lower value of $\sigma_{8}$ than suggested by the Planck-CMB analysis in order to match the observed redshift distribution of the XXL-100 (Fig. 1, right).

\section{Modeling the X-ray sky}

\subsection{The X-ray Universe as seen through a super computer}

We use the Magneticum Pathfinder (www.magneticum.org) hydro-dynamical cosmological simulation set to investigate the footprint of AGNs in clusters for future X-ray 

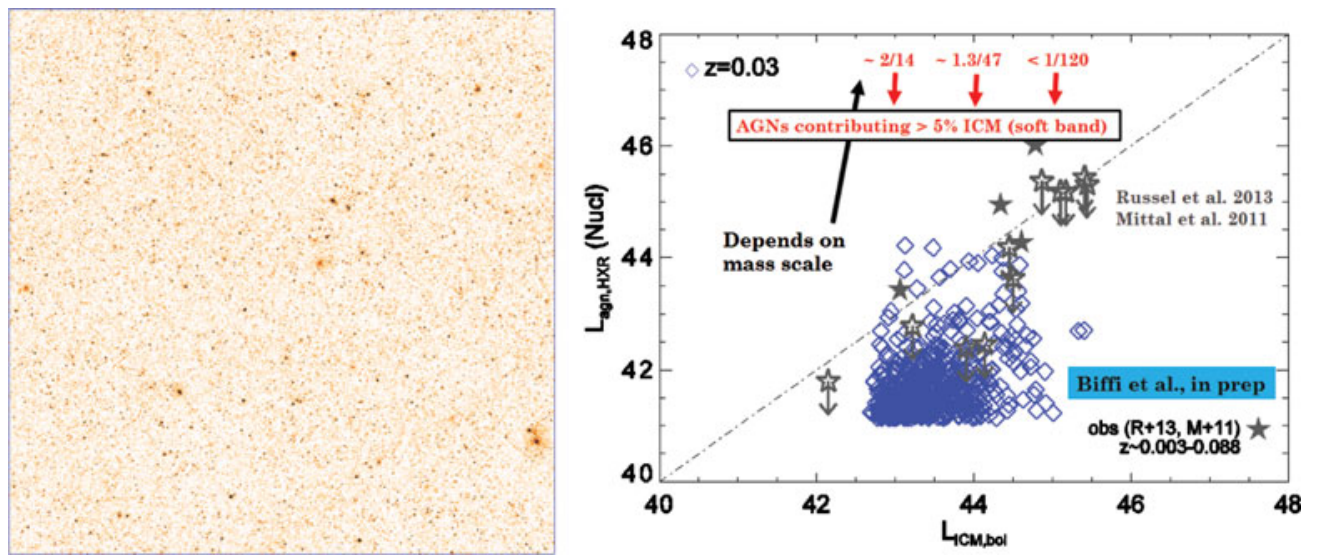

Figure 2. Left: $5 \times 5$ degree wide lightcone constructed from the cosmological, hydrodynamical simulation containing the ICM and AGN contribution up to a redshift $z=2$ for a typical $1.6 \mathrm{ks}$ field in the eROSITA all-sky survey. Right: X-ray luminosity of the central AGN versus the ICM bolometric luminosity from the simulations (blue diamonds) and comparison to observational detections and upper limits (grey asterisks).

missions like eROSITA and ATHENA. The simulations treat metal-dependent radiative cooling, heating from a uniform time-dependent ultraviolet background, star formation and the chemo-energetic evolution of the stellar population as traced by SNIa, SNII and AGB stars with the associated feedback processes, as well as formation and evolution of super-massive black holes and the associated quasar and radio-mode feedback processes (see Hirschmann et al. 2014 for details).

The PHOX code (Biffi et al. 2013) generates the X-ray emission from the simulations, taking the intra cluter medium (ICM) as well as the active galactic nuclei (AGN) into account. For the later, we convert the bolometric luminosities $\mathrm{L}_{\mathrm{bol}}$ of the AGN into SXR and HXR luminosities assuming the bolometric corrections proposed by Marconi et al. (2004), which can be approximated by the following third-degree polynomial fits

$$
\begin{aligned}
\log \left(\mathrm{L}_{\mathrm{HXR}} / \mathrm{L}_{\mathrm{bol}}\right) & =-1.54-0.24 \mathcal{L}-0.012 \mathcal{L}^{2}+0.0015 \mathcal{L}^{3} \\
\log \left(\mathrm{L}_{\mathrm{SXR}} / \mathrm{L}_{\mathrm{bol}}\right) & =-1.65-0.22 \mathcal{L}-0.012 \mathcal{L}^{2}+0.0015 \mathcal{L}^{3}
\end{aligned}
$$

with $\mathcal{L}=\log \left(\mathrm{L}_{\mathrm{bol}} / \mathrm{L}_{\odot}\right)-12$. Here we mimic the observed scatter in these relations by adding a random scatter of 0.1 decades to the SXR and HXR luminosities. Then we construct an intrinsic redshifted power-law spectrum

$$
A(E)=[K(1+z)][E(1+z)]^{-\alpha}(1+z)^{-1},
$$

where $K$ is the normalization, $\alpha$ the photon index and $z$ the redshift of the source. In this approach, the obtained photon index $\alpha$ that reasonably reflects the observed Gaussian distribution in the range 1.2-2.8, which peaks around $\sim 2$ (e.g. Zdziarski et al. 2000)

Observations suggest evidence for AGN to present obscuring material (i.e. the torus) in the vicinity of the central $\mathrm{BH}$ and absorbing the emitted radiation. Therefore, in our implementation, the specific value of the obscurer column-density $\left(N_{h}\right)$ is assigned to each AGN source in a probabilistic way, by assuming the estimated column-density distribution of the obscurer obtained by Buchner et al. (2014) (top-left panel of their Fig. 10) from a sample of 350 X-ray AGN in the 4 Ms Chandra Deep Field South. Within the PHOX code, we include this in the construction procedure of the X-ray emission model from BH (AGN-like) sources. The resulting absorbed SXR and HXR luminosity functions are in good agreement with the observed ones (Biffi et al. in prep.) 
This allows to finally produce mock observations (Fig. 2, left) containing self consistently the contributions of ICM and AGN for eROSITA like exposure as well as to study in detail the contamination of the cluster X-ray emission by the presence of AGN in the central part as well as in satellite galaxies due to their X-ray footprint (Fig. 2, right). We find that the number of AGN found in member galaxies contributing significantly to the X-ray luminosity of the ICM strongly depends on the mass of the cluster. Our simulations contain typically 14 well resolved member galaxies in low mass, group like systems with $\mathrm{L}_{\mathrm{bol}} \approx 10^{43} \mathrm{erg} / \mathrm{s}$, of which on average two are hosting AGN with X-ray luminosities of at least $5 \%$ of the host halo. For galaxy clusters with $\mathrm{L}_{\mathrm{bol}} \approx 10^{45} \mathrm{erg} / \mathrm{s}$ however we find on average more than 100 well resolved member galaxies, of which typically one (but sometimes even none) hosts an AGN with an X-ray luminosity of at least $5 \%$ of the host halo.

\subsection{The survival and destruction of Galactic X-ray coronae in groups and clusters}

In Vijayaraghavan \& Ricker (2015), we present the results of $N$-body + hydrodynamic simulations of 26 galaxies in an isolated group of mass $3 \times 10^{13} \mathrm{M}_{\odot}$ and 152 galaxies in an isolated cluster of mass $1.2 \times 10^{14} \mathrm{M}_{\odot}$. These simulations were performed with the FLASH code, with minimum spatial resolution of up to $1.6 \mathrm{kpc}$ and $10^{6} \mathrm{M}_{\odot}$ particles. Galaxies in these simulations are NFW spheres with a collisionless dark matter component and hot gas component with $10 \%$ of the total mass of the galaxy.

Figure 3 shows ram pressure stripping in action for a range of group and cluster galaxies. Stripped gas trails galaxies in their orbits, forming shear instabilities at the ISMICM interface, before eventually dissipating within the ICM. By $\sim 3$ Gyr, most galaxies have lost all their gas. The amount of gas removed depends on the mass of the galaxy and the host. Galaxies in the less massive group have smaller velocities and experience weaker ram pressure compared to galaxies in the massive, high velocity dispersion cluster. Group galaxies therefore lose gas at a slower rate than cluster galaxies. In a given environment, more massive galaxies, with larger gravitational restoring forces, are more resistant to ram pressure stripping. A more detailed quantitative analysis of this process is described in Vijayaraghavan \& Ricker (2015).

We generated synthetic Chandra X-ray observations with 40 and 400 ks exposure times of the simulated group and cluster, including their galaxies (an example is illustrated in Figure 4). Galaxy wakes and tails are visible up to $\sim 1$ Gyr in the $40 \mathrm{ks}$ image, and their surviving central coronae up to $\sim 2$ Gyr, albeit at low significance levels above the cluster background. Galactic tails are visible up to 2 Gyr in the $400 \mathrm{ks}$ images. Galactic coronal emission can be detected observationally in short exposure observations by stacking regions around individual cluster galaxies identified in other wavebands. There is an excess in stacked galactic surface brightness profiles at $r \lesssim 10$ arcsec in group and cluster galaxies up to 2.38 Gyr in the low energy $0.1<E<1.2 \mathrm{keV}$ band. This excess persists on subtracting the correspondingly stacked emission centered on points diametrically opposite known galaxy centers. The X-ray emission from cluster galaxies declines faster than that of group galaxies, since galaxies in massive clusters experience stronger ram pressure. Additionally, the emission from galaxies at small galaxy-centric radii manifests itself in measurements of the hardness ratio $\left(E_{\mathrm{hard}} / E_{\mathrm{soft}}\right)$, as a noticeable decrease in hardness ratio in the regions with significant galactic emission. These results are illustrated in Figure 5 .

Magnetic fields in the ICM also affect the loss of galactic coronal gas, and galaxies themselves affect the overall strength and morphology of ICM magnetic fields (Vijayaraghavan \& Ricker, in prep.). Stripped galaxy tails can be supported by magnetic pressure. Magnetic fields lines aligned with galaxy tails suppress shear instabilities and 

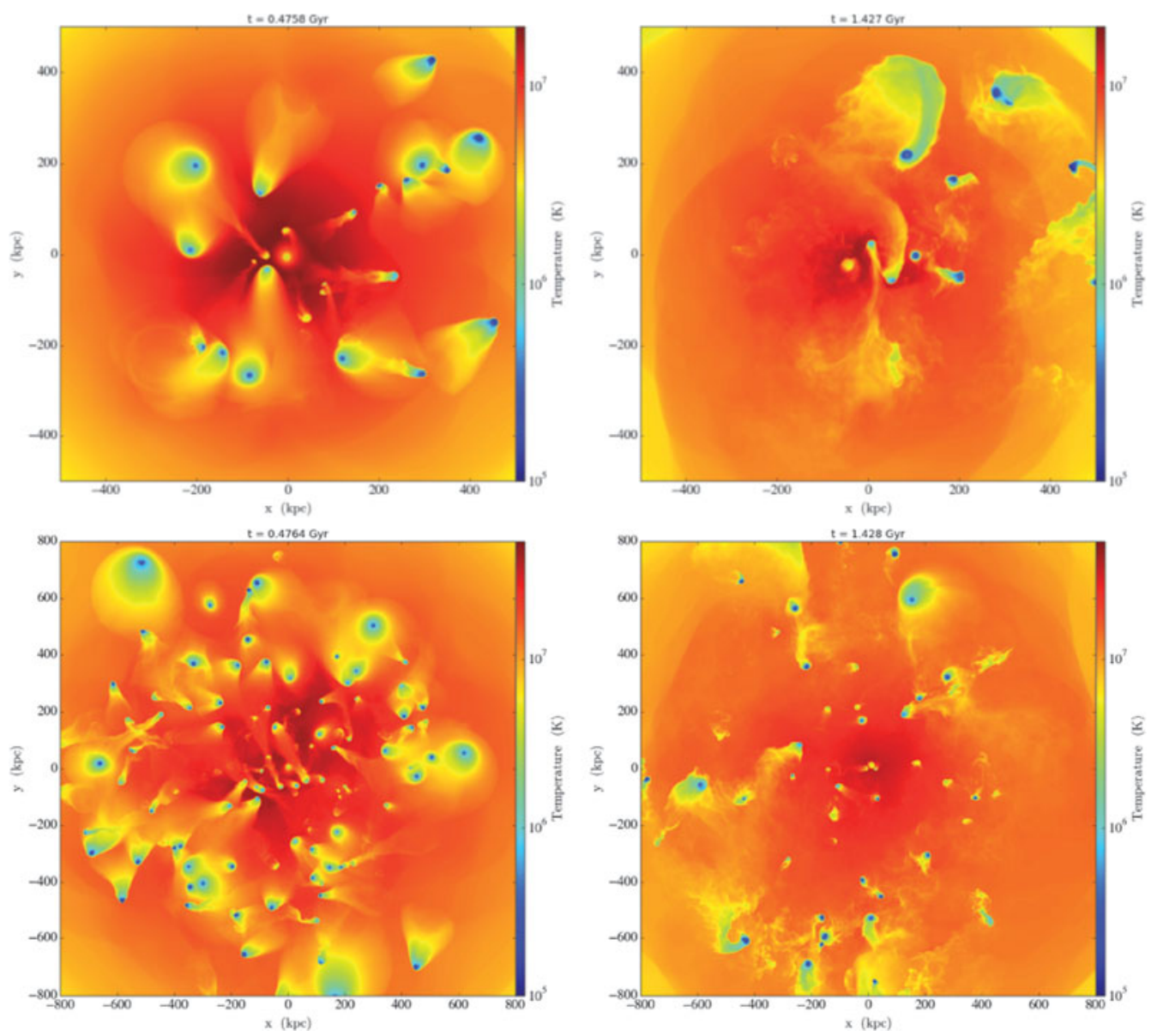

Figure 3. The evolution of gas in the group (top) and cluster (bottom) galaxies, as seen in maps of emission measure-weighted temperature. Galaxies are stripped of their gas by the ICM, and the stripped gas trails galaxies in their orbits in the form of wakes before mixing with the ICM.

mixing with the ICM. Magnetic fields, draped along the leading edges of galactic coronae and supporting stripped tails, can suppress thermal conduction between the ISM and ICM and thereby suppress gas loss due to evaporation. Galaxy motions drive turbulence within the ICM, increasing the amplitude of magnetic fields and resulting in a more tangled configuration.

The long survival timescales of observed galactic coronae therefore implies that additional physical processes, potentially outflows from the galaxies or radiative cooling, is likely responsible in replenishing stripped gas. Existing samples (e.g., the ACCEPT sample, Cavagnolo et al. 2009; the XCS, Mehrtens et al. 2012) and upcoming X-ray surveys of clusters (e.g., with eROSITA) can be used to systematically study the effect of the cluster environment on circumgalactic coronae by stacking galactic X-ray emission.

\section{Near-future prospects: $e$ ROSITA and its all-sky survey}

Starting 2017, the eROSITA instrument onboard the SRG satellite will be positioned at L2 and scan the sky during four years in X-rays at $0.3-10 \mathrm{keV}$ (Predehl et al. 2014). 

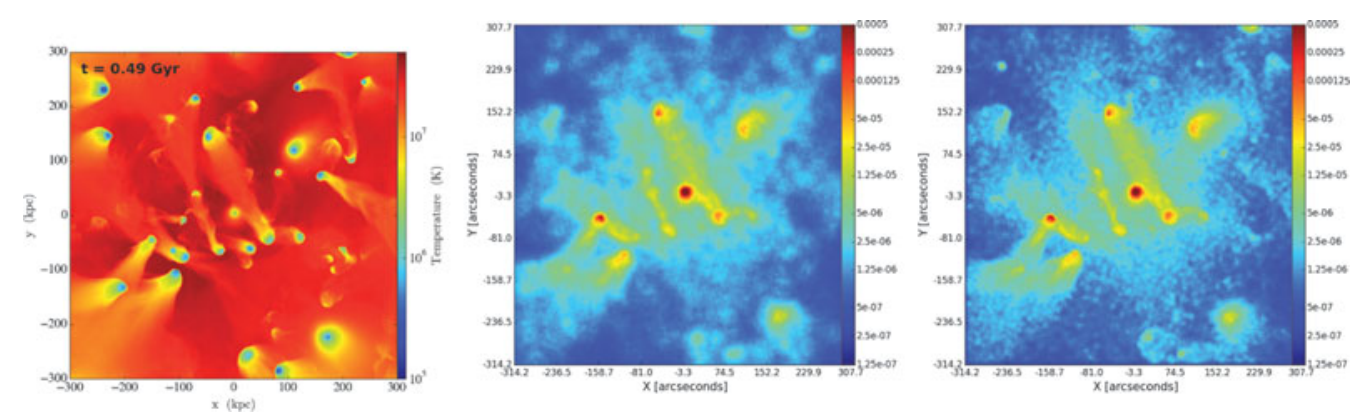

Figure 4. Left: Projections of the emission measure weighted temperature of the cluster's central $600 \mathrm{kpc}^{2}$ region at $t=0.47 \mathrm{Gyr}$. Center and right: Mock $40 \mathrm{ks}$ and $400 \mathrm{ks}$ images of the central region, after accumulative smoothing. The colors correspond to the photon flux in units of counts second ${ }^{-1} \operatorname{arcsecond}^{-2}$.
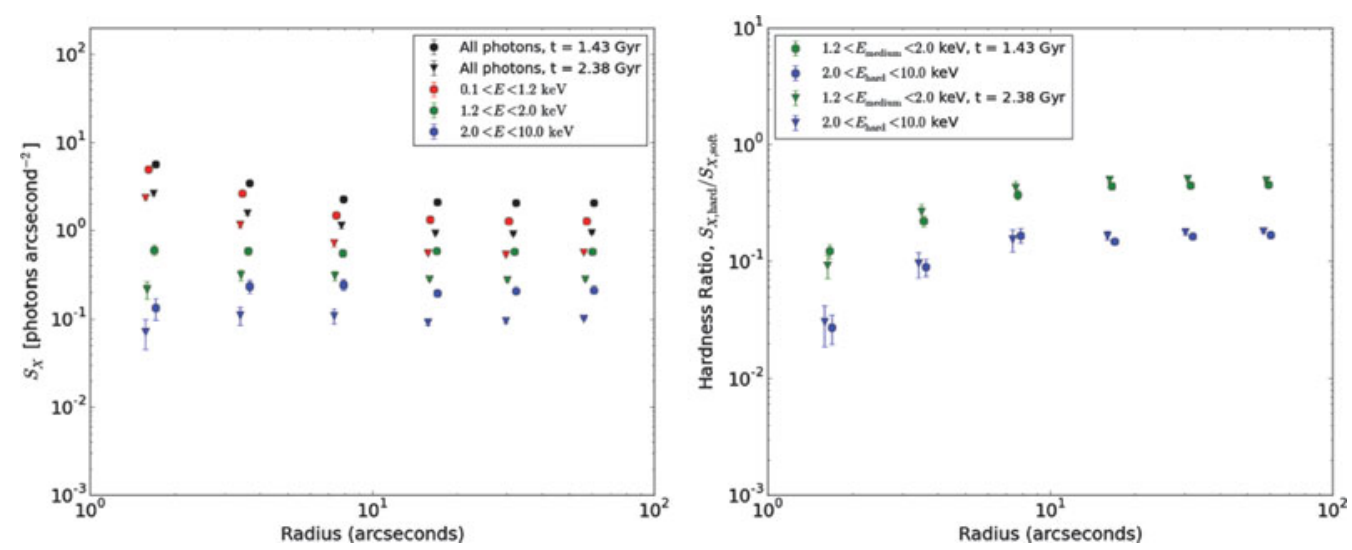

Figure 5. Left: Stacked, background subtracted, radial profile for cluster galaxies (that are at least $200 \mathrm{kpc}$ from the cluster center in projection). Right: Hardness ratio profile.

Every six months a deeper X-ray image of the full sky will be acquired at $\sim 28 \operatorname{arcsec}$ angular resolution (soft band). Sensitivities will be 30 times deeper than the ROSAT allsky survey in the soft band, typically used for cluster detection (Fig. 6 and Merloni et al. 2012). About 3 million AGN are expected, while 80 to 100 thousand galaxy clusters will be discovered as extended X-ray sources over the extragalactic sky after survey completion. eROSITA will realize the census of all massive clusters up to $z \sim 1$ and probe the large-scale structure down to the massive group regime (Fig. 7, left), such frontiers being pushed even further in the two deeply exposed ecliptic polar areas. Survey data will enable precise characterization of a fraction of the sources (locations, sizes, morphology, etc.) with about 11,000 systems providing more than $500 \mathrm{X}$-ray counts and 1,700 having gas temperatures measurable down to $10 \%$ accuracy (Borm et al. 2014).

A substantial fraction of eROSITA clusters will not deliver enough X-ray counts to provide accurate mass measurements from survey data itself, those being the main input of cosmological analyses. Alternative analysis routes include transferring the cosmological analysis in the X-ray observable domain. By doing so, the calculation of individual cluster masses is avoided, and self-consistently enclosed in calibrated scaling relations linking mass and X-ray observables. Clerc et al. (2012a) proposed to model the population of $\mathrm{X}$-ray clusters in the count-rate/hardness ratio plane, possibly adding the redshift of clusters as a supplementary piece of information. The method was applied to a simulated 


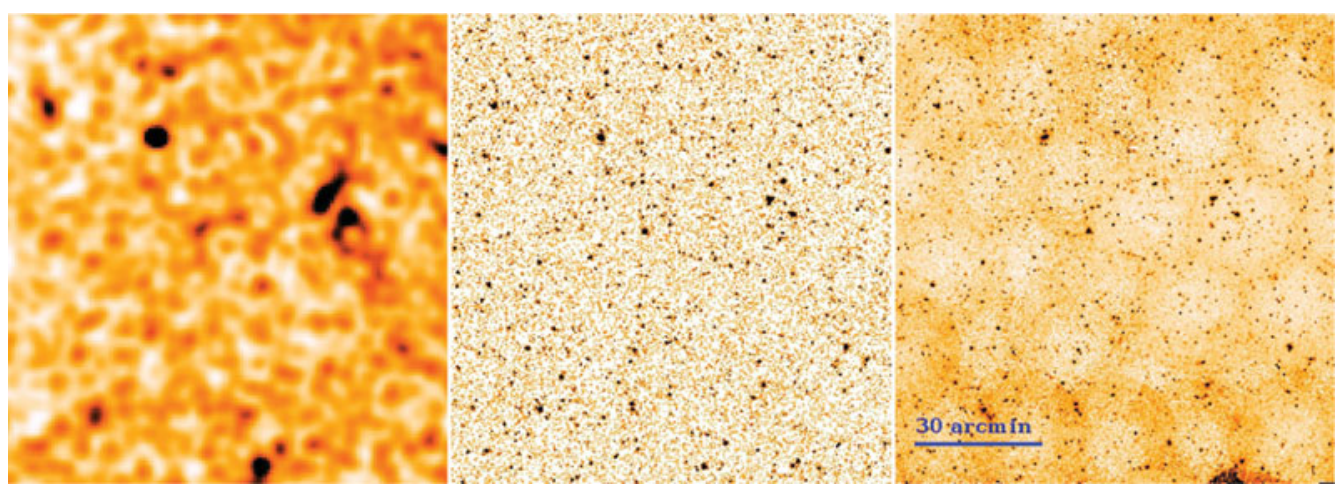

Figure 6. Comparison of a same field in the ROSAT all-sky survey (left, in the [0.1-2.4] keV band), the eROSITA 4-year survey (centre, simulated eRASS:8 [0.5-2] keV) and the XMM-XXL survey (right, $[0.5-2] \mathrm{keV}$ ). The eRASS:8 soft-band will probe 30 times deeper sensitivities than ROSAT and reach extended source fluxes of $4 \times 10^{-14} \mathrm{ergs. \textrm {s } ^ { - 1 }} . \mathrm{cm}^{-2}$ over the entire sky.
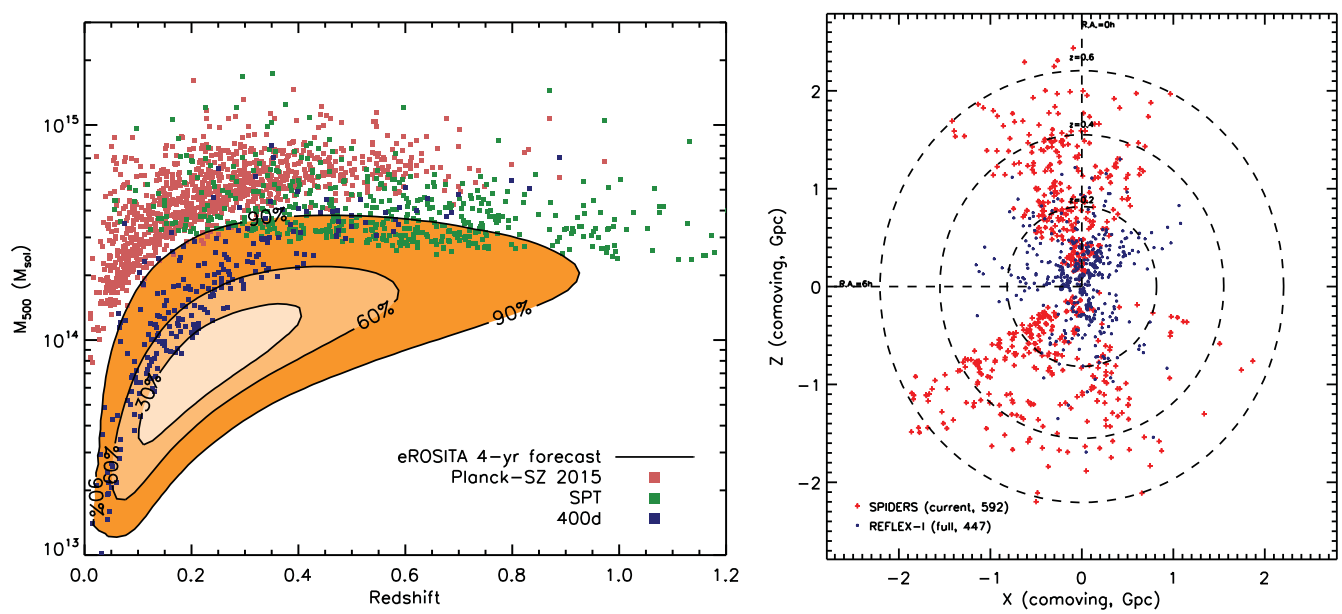

Figure 7. Left: expected redshift-mass distribution of eROSITA galaxy clusters. The contours enclose $30,60,90 \%$ of the population of $\sim 10^{5}$ systems detectable after four years of survey. Colored points: Burenin et al. (2007), Bleem et al. (2015), Planck Collaboration et al. (2015b). Right: Tridimensional map of X-ray galaxy clusters from the SPIDERS survey (red) after its first year of operation. Blue points are the REFLEX-I clusters (Böhringer et al. 2004).

eROSITA population of galaxy clusters to forecast the cosmological constraining power of the method (Fig. 8, left and Clerc et al. 2012b). Pillepich et al. (2012) show detailed cosmological forecasts for the eROSITA sample, combining number counts and power spectrum experiments, and demonstrate their complementarity in cosmological analyses (see also e.g. Sartoris et al. 2010 and Fig. 8, right).

Such forecasts assume the availability of redshifts for each individual system discovered by eROSITA. The follow-up of eROSITA clusters (Merloni et al. 2012) involves observations with 4MOST at ESO (starting 2020, De Jong et al. 2014) in the Southern hemisphere and with the SDSS telescope in the North. The SPectroscopic IDentification of eROSITA Sources (SPIDERS) is a programme part of SDSS-IV launched in 2014 (Clerc et al. in prep.) and is measuring the distance of X-ray detected galaxy clusters via optical spectroscopy of its galaxy members. Fig. 7 (right) summarizes the current status of the programme, currently focused on observing ROSAT clusters (the CODEX 

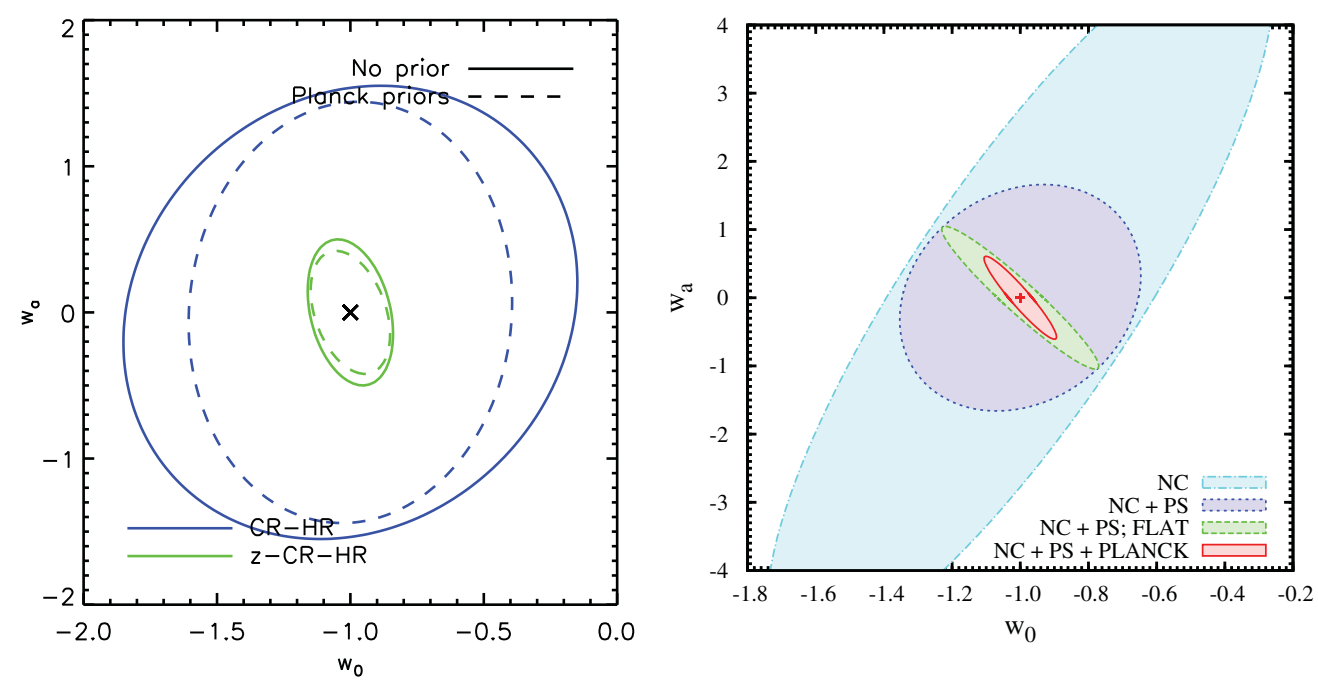

Figure 8. Predicted $68 \%$ confidence level constraints on the dark energy EoS parameters $\left(w_{0}\right.$, $\left.w_{a}\right)$. Left: for the 4-year eROSITA clusters, based on the X-ray count-rate (CR), hardness-ratio $(\mathrm{HR})$ and redshift $(z)$ cluster number counts assuming a flat Universe (details in Clerc et al. $2012 \mathrm{~b})$. Right: for the clusters from the three WFXT surveys with enough X-ray photons to calculate a mass. Generally, combining number counts (NC) and power-spectrum (PS) analyses improves those constraints (details in Sartoris et al. 2012). In both cases "Planck" correspond to Planck CMB priors: they break degeneracies by constraining the geometry of the Universe.

sample, Finoguenov et al., in prep.): SPIDERS has already begun mapping the large-scale structure out to large redshifts $(z \sim 0.6)$ through its most massive constituents.

Sect. 2 discussed the importance of an accurate cluster mass calibration and the role played by follow-up observations in this respect, especially in X-rays (Chandra and $X M M)$. Intense follow-up campaigns of $\mathcal{O}\left(10^{5}\right)$ object samples will be unfeasible. Three X-ray surveys - complementary in terms of sensitivity and sky coverage - with the WFXT (e.g. Murray et al. 2010 ) can provide $\sim 2.5 \times 10^{5}$ clusters and measurements of the lowscatter mass proxy $Y_{X}$ (Kravtsov et al. 2006) for $\sim 2 \times 10^{4}$ clusters, up to redshift $z \sim 2$. A wide all sky survey will detect extended sources in the [0.5-2] keV energy band down to a flux $0.5 \times 10^{-14} \mathrm{erg} . \mathrm{s}^{-1} . \mathrm{cm}^{-2}$, while a deep survey will reach $0.01 \times 10^{-14} \mathrm{erg} . \mathrm{s}^{-1} . \mathrm{cm}^{-2}$. For objects with a flux limit 30 times brighter than the cluster detection limit, measurements of the mass proxies for a large fraction of clusters in the survey mode will be possible, using $\gtrsim 6000$ net counts per cluster (Sartoris et al. 2010 and Fig. 8).

\section{Summary}

The cosmological constraints reviewed in Sect. 2 are based on relatively small samples (a few hundred galaxy clusters or less), however they are competitive with respect to other cosmological probes. Upcoming surveys, in particular and starting 2017 the eROSITA all-sky survey, will transform the field by bringing these numbers up to $\sim 10^{5}$. Their cosmological exploitation and success will result from a combination of: a self-calibration of the halo mass-observables in these samples; external calibrations using samples adequately chosen in a parameter space spanning ranges of halo masses, redshifts, relaxation states, environments, etc.; multiple methods to measure a cluster mass (from X-rays, weak-lensing, spectroscopic observations, etc.) ; numerical simulations faithfully reproducing the observed X-ray sky, both at largest scales (the hot cosmic web) and at the 
cluster scale (the gas in the halos). By the end of the 2020s, cosmological models will be tightly constrained by current and near-future experiments, in particular X-ray surveys. Those will raise new questions on the origin, the cycle and the fate of the hot baryons in clusters and groups, to be answered by next-generation observatories such as ATHENA (e.g. Nandra et al. 2013; Barcons et al. 2015).

\section{References}

Albrecht, A., Bernstein, G., Cahn, R., et al. 2006, arXiv:astro-ph/0609591

Allen, S. W., Rapetti, D. A., Schmidt, R. W., et al. 2008, MNRAS, 383, 879

Allen, S. W., Evrard, A. E., \& Mantz, A. B. 2011, ARAA, 49, 409

Amendola, L. 2000, Phys. Rev. D, 62, 043511

Bahcall, N. A. \& Bode, P. 2003, ApJ (Letters), 588,L1

Barcons, X., Nandra, K., Barret, D., et al. 2015, Journal of Physics Conference Series, 610, 012008

Biffi, V., Dolag, K., Böhringer, H. 2013, MNRAS, 428, 1395

Bleem, L. E., Stalder, B., de Haan, T., et al. 2015, ApJS, 216, 27

Böhringer, H., Schuecker, P., Guzzo, L., et al. 2004, A\&A, 425, 367

Böhringer, H., Chon, G., \& Collins, C. A. 2014, A\&A, 570, A31

Borgani, S., Rosati, P., Tozzi, P., et al. 2001, ApJ, 561, 13

Borm, K., Reiprich, T. H., Mohammed, I., \& Lovisari, L. 2014, A\&A A, 567, A65

Brodwin, M., Ruel, J., Ade, P. A. R., et al. 2010, ApJ, 721, 90

Buchner, J., Georgakakis, A., Nandra, K., et al. 2014, A\&AA, 564, A125

Burenin, R. A., Vikhlinin, A., Hornstrup, A., et al. 2007, ApJS, 172, 561

Caldwell, R. R. \& Kamionkowski, M. 2009, Annual Review of Nuclear and Particle Science, 59, 397

Cavagnolo, K. W., Donahue, M., Voit, G. M., \& Sun, M. 2009, ApJS, 182, 12

Clerc, N., Pierre, M., Pacaud, F., \& Sadibekova, T. 2012a, MNRAS, 423, 3545

Clerc, N., Sadibekova, T., Pierre, M., et al. 2012b, MNRAS, 423, 3561

Costanzi, M., Villaescusa-Navarro, F., Viel, M., et al. 2013, JCAP, 12, 012

Dvali, G., Gabadadze, G., \& Porrati, M. 2000, Phys. Lett. B, 485, 208

Eke, V. R., Cole, S., Frenk, C. S., Patrick Henry, J., 1998, MNRAS, 298, 1145

Ettori, S., Morandi, A., Tozzi, P., et al. 2009, A\&A, 501, 61

Henry, J. P. 2000, ApJ, 534, 565

Henry, J. P., Evrard, A. E., Hoekstra, H., Babul, A., \& Mahdavi, A. 2009, ApJ, 691, 1307

Hinshaw, G., Larson, D., Komatsu, E., et al. 2013, ApJS, 208, 19

Hirschmann, M., Dolag, K., Saro, A., et al. 2014,MNRAS, 442, 2304

Hu, W. \& Sawicki, I. 2007, Phys. Rev. D, 76, 064004

de Jong, R. S., Barden, S., Bellido-Tirado, O., et al. 2014, Proc. SPIE, 9147, 91470M

Kravtsov, A. V., Vikhlinin, A., \& Nagai, D. 2006, ApJ, 650, 128

Mantz, A. B., Allen, S. W., Morris, R. G., et al. 2014, MNRAS, 440, 2077

Mantz, A. B., von der Linden, A., Allen, S. W., et al. 2015a, MNRAS, 446, 2205

Mantz, A. B., Allen, S. W., Morris, R. G., et al. 2015b, MNRAS, 449, 199

Marconi, A., Risaliti, G., Gilli, R., et al. 2004, MNRAS, 351, 169

Mehrtens, N., Romer, A. K., Hilton, M., et al. 2012, MNRAS, 423, 1024

Merloni, A., Predehl, P., Becker, W., et al. 2012, arXiv:1209.3114

Mortonson, M. J., Hu, W., \& Huterer, D. 2011, Phys. Rev. D, 83, 023015

Movahed, M. S., Farhang, M., \& Rahvar, S. 2009, International Journal of Theoretical Physics, 48,1203

Mullis, C. R., Rosati, P., Lamer, G., et al. 2005, ApJ (Letters), 623, L85

Murray, S. S., Giacconi, R., Ptak, A., et al. 2010, Proc. SPIE, 7732, $77321 \mathrm{~W}$

Nandra, K., Barret, D., Barcons, X., et al. 2013, arXiv:1306.2307

Perlmutter, S., Aldering, G., Goldhaber, G., et al. 1999, ApJ, 517, 565

Pierpaoli, E., Scott, D., \& White, M. 2001, MNRAS, 325, 77 
Pillepich, A., Porciani, C., \& Reiprich, T. H. 2012, MNRAS, 422, 44

Planck Collaboration, Ade, P. A. R., Aghanim, N., et al. 2015a, arXiv:1502.01589

Planck Collaboration, Ade, P. A. R., Aghanim, N., et al. 2015b, A\&AA, 581, A14

Predehl, P., Andritschke, R., Becker, W., et al. 2014, Proc. SPIE, 9144, 91441T

Ratra, B. \& Peebles, P. J. E. 1988, Phys. Rev. D, 37, 3406

Rest, A., Scolnic, D., Foley, R. J., et al. 2014, ApJ, 795, 44

Riess, A. G., Filippenko, A. V., Challis, P., et al. 1998, AJ, 116, 1009

Rosati, P., Tozzi, P., Gobat, R., et al. 2009, A\& $A, 508,583$

Samushia, L., Reid, B. A., White, M., et al. 2014, MNRAS, 439, 3504

Sartoris, B., Borgani, S., Fedeli, C., et al. 2010, MNRAS, 407, 2339

Sartoris, B., Borgani, S., Rosati, P., \& Weller, J. 2012, MNRAS, 423, 2503

Schuecker, P., Böhringer, H., Collins, C. A., \& Guzzo, L. 2003, A\&AA, 398, 867

Seljak, U. 2002, MNRAS, 337, 769

Skordis, C. 2009, Classical and Quantum Gravity, 26, 143001

Sheth, R. K. \& Tormen, G. 1999, MNRAS,308, 119

Sotiriou, T. P. \& Faraoni, V. 2010, Reviews of Modern Physics, 82, 451

Tinker, J., Kravtsov, A. V., Klypin, A., et al. 2008, ApJ, 688, 709

Tsujikawa, S. 2010, arXiv:1004.1493

Viana, P. T. P., \& Liddle, A. R. 1999, MNRAS, 303, 535

Vijayaraghavan, R. \& Ricker, P. M. 2015, MNRAS, 449, 2312

Vikhlinin, A., Burenin, R. A., Ebeling, H., et al. 2009b, ApJ, 692, 1033

Vikhlinin, A., Kravtsov, A. V., Burenin, R. A., et al. 2009c, ApJ, 692, 1060

Voevodkin, A. \& Vikhlinin, A. 2004, ApJ, 601, 610

Zdziarski, A. A., Poutanen, J., Johnson, W. N. 2000,ApJ, 542, 703 